\title{
Fine structure of erythrocytic stages of a Plasmodium tropiduri-like malaria parasite found in the lizard Kentropyx calcarata (Teiidae) from north Brazil
}

\author{
Ilan Paperna ${ }^{1}$ and Ralph Lainson ${ }^{2}$ \\ ${ }^{1}$ Department of Animal Sciences, Faculty of Agriculture of the Hebrew University of Jerusalem, Rehovot 76-100, Israel; \\ ${ }^{2}$ Department of Parasitology, The Instituto Evandro Chagas, Avenida Almirante Barroso 492, Belém 66090-000, Pará, Brazil
}

Key words: Plasmodium, Kentropyx calcarata, Brazil, merogony, gametocytes, ultrastructure

\begin{abstract}
The fine structure is described of the merogonic stages and gametocytes of a Plasmodium tropiduri Aragão et Neiva, 1909-like parasite infecting the teiid lizard Kentropyx calcarata Spix from North Brazil. The trophozoites are bordered by two membranes, and with growth a pellicle is formed by the addition of an inner, thick double layer and fragmented membrane. The same type of inner membrane occurs in the pellicle of the merozoites differentiating from the meronts. Merozoites contained a large electron-dense body, sometimes seen to be embraced by a tubular mitochondrion with a dense matrix. Micro- and macrogametocytes are bounded by a double membrane, closely apposed by the detached wall of the parasitophorous vacuole. Both contain osmiophilic bodies. The microgametocyte contains an electron-dense aggregate, and the macrogametocyte has a large mitochondrion and a complex of tubuli and cisternae. These features are compared with those described in other malarial parasites.
\end{abstract}

Specimens of Kentropyx calcarata Spix from Pará State, north Brazil were found to harbour two malarial parasites of very different morphology as seen in Giemsa-stained blood films (Lainson et al. 2001). One, Plasmodium kentropyxi Lainson, Landau et Paperna, 2001 possesses large elongate gametocytes and meronts producing 30-40 merozoites, both occupying a lateral position in the erythrocyte. The other is morphologically very similar to Plasmodium tropiduri Aragão et Neiva, 1909, with small, rounded gametocytes and meronts producing only 4-14 merozoites, both consistently in a polar or lateropolar position in the red blood cell.

Plasmodium tropiduri was first described by Aragão and Neiva (1909) from the iguanid lizard Tropidurus torquatus from Minas Gerais, Brazil. Similar parasites have been described in the teiid Ameiva ameiva (Walliker 1966), and the skink Mabuya mabouya (Lainson and Shaw 1969) both from Brazil, and from various Anolis species (Iguanidae) from Panama (Telford 1979). It is questionable as to whether or not these plasmodia are conspecific with $P$. tropiduri sensu stricto from the type host $T$. torquatus. Scorza (1971) made an ultrastructural study of the latter parasite from Venezuela, while the fine structure of other reptilian Plasmodium species has been described by Aikawa and Jordan (1968) and Moore and Sinden (1974). A comparative account of the ultrastructure of gametocytes of reptilian, avian and mammalian Plasmodium spp. has been given by Aikawa et al. (1969). In this paper we describe the fine structure of the merogonic stages and gametocytes of the $P$. tropiduri-like parasite recently encountered in the teiid K. calcarata from North Brazil.

\section{MATERIALS AND METHODS}

Kentropyx calcarata were collected in Capanema $\left(1^{\circ} 12^{\prime} \mathrm{S}\right.$, $\left.47^{\circ} 11^{\prime} \mathrm{W}\right)$, Pará, North Brazil. Specimens with only stages of the $P$. tropiduri-like parasite in Giemsa-stained blood films were sacrificed and pieces of clotted blood and tissues (liver, lung and spleen) were fixed in $2.5 \%$ glutaraldehyde in cacodylate buffer $(0.1 \mathrm{M}, \mathrm{pH} 7.4)$ for 24 hours at $4^{\circ} \mathrm{C}$. They were then rinsed repeatedly in the same buffer, post-fixed in $1.0 \%$ osmium tetroxide in the same buffer for one hour and, after rinsing in the buffer, dehydrated in graded ethyl alcohols and embedded in Agar 100 medium (Agar Scientific, Ltd., UK). Thin sections, cut on a Reichert "Ultracut" microtome with a diamond knife, were stained on grids with uranyl acetate and lead citrate, and examined with a JEOL 100CX transmission electron microscope (TEM).

\section{RESULTS}

\section{Trophozoites}

Trophozoites were bordered by two conspicuous membranes, the outer one being the thickest. Between these there could be traced faint outlines of an additional lamella. The cytoplasm of the trophozoites contained scattered aggregates of ribosomes, bundles of subpellicular microtubules, a mitochondrion and an inclusion with a bilamellar border (food vacuole?). The endoplasmic reticulum (ER) appeared adjacent to the 


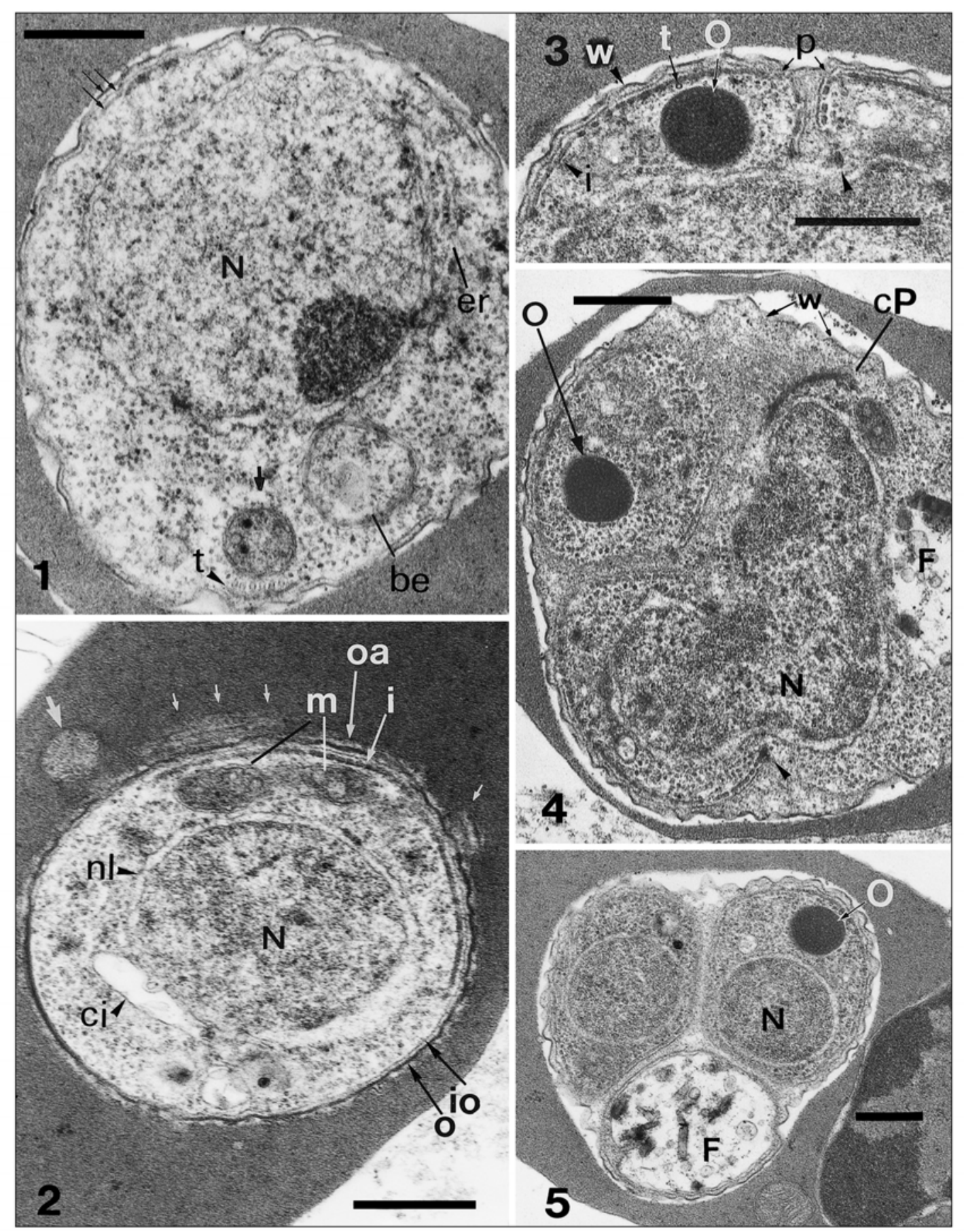

Figs. 1-5. Trophozoites and emerging merozoites of a Plasmodium tropiduri-like parasite of the lizard Kentropyx calcarata. Fig. 1. A trophozoite, surrounded by two membranes and intervening lamella (small arrows), showing a nucleus (N), endoplasmic reticulum (er), mitochondrion (bold arrow), an array of microtubules ( $\mathrm{t}$ ) and an inclusion with a bilaminar wall (be). Fig. 2. Trophozoite forming a pellicle consisting of an outer wall (o), inner membrane (io), fragmented wall (i) and additional superficial layer (oa). Clefts (thin arrow) and a mitochondrion (bold arrow) can be seen in the erythrocyte (ci - cisterna, $\mathrm{m}-$ mitochondria, $\mathrm{N}$ - nucleus, $\mathrm{nl}$ - nucleolemma). Fig. 3. Emerging merozoite rudiments within the meront wall membrane (w). They are invested by an outer pellicular membrane (p), an inner fragmented wall (i), and underlying microtubules ( $t$ ), and contain an osmiophilic-granular body $(\mathrm{O})$; arrowhead - junction element. Fig. 4. Meront with emerging merozoite (cP centriolar plaque, $\mathrm{F}$ - food vacuole with pigment, $\mathrm{N}$ - nucleus, $\mathrm{O}$ - osmiophilic-granular body, arrowhead - junction element). Fig. 5. Merozoites emerging above the meront residuum (for abbreviations see Fig. 4). Scale bars $=0.5 \mu \mathrm{m}$. 


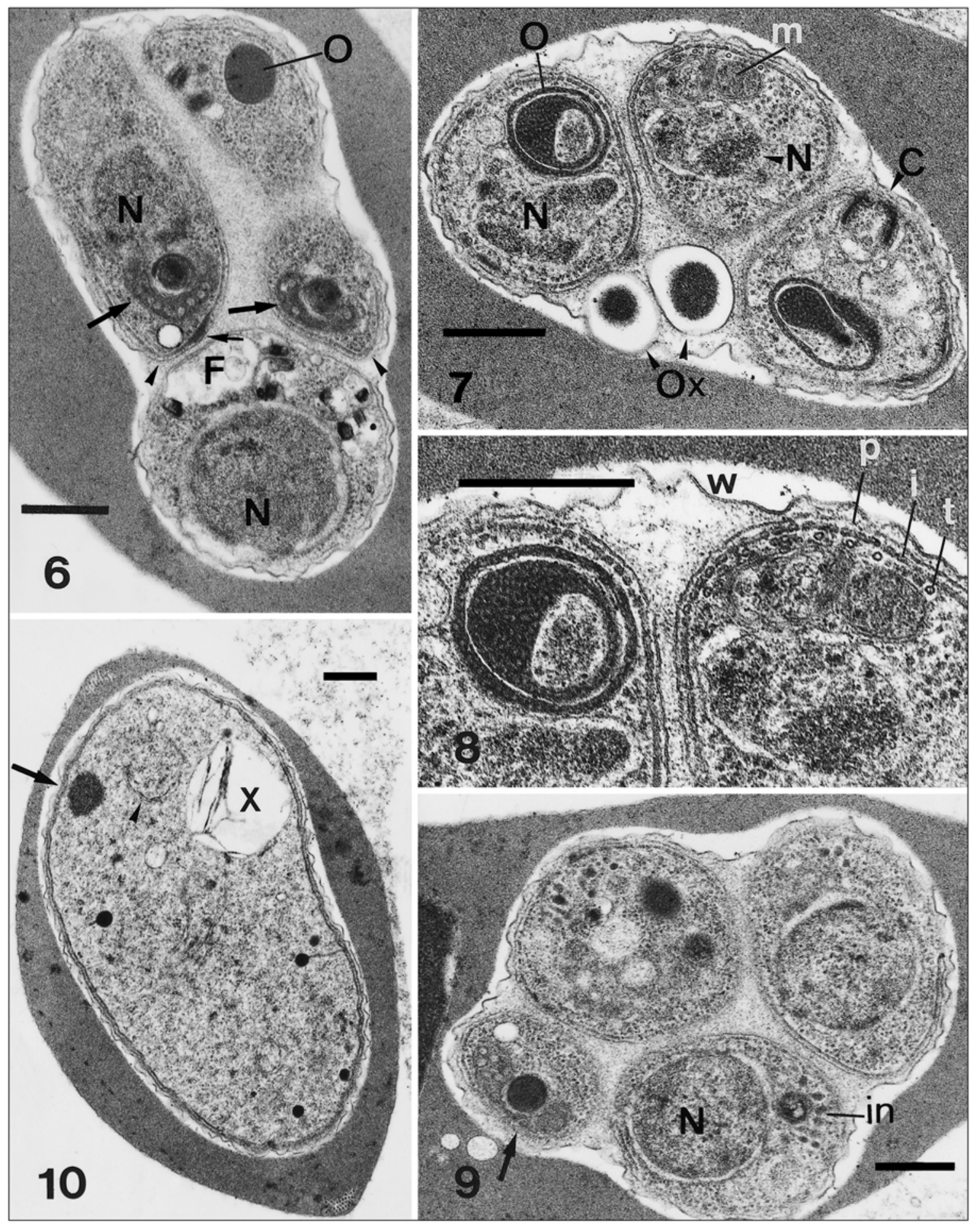

Figs. 6-10. A Plasmodium tropiduri-like parasite of the lizard Kentropyx calcarata: differentiating merozoites and a microgametocyte. Fig. 6. Merozoites showing mitochondria with electron-dense matrix (bold arrows), embracing an osmiophilic-granular body (O). There is a meront residuum with a nucleus $(\mathrm{N})$ and food vacuole containing pigment (F). (Arrowhead - merozoite apical zone, fine arrow - thickening of the pellicle). Fig. 7. Cross-section of merozoites showing nuclei $(\mathrm{N})$, disaggregation of large intracellular $(\mathrm{O})$ and extracellular $(\mathrm{Ox})$ osmiophilic granular bodies, cytostome $(\mathrm{C})$ and mitochondria (m). Fig. 8. Enlargement from Fig. 7 (for abbreviations see Figs. 2, 3). Fig. 9. Cross-section of a developing merozoite showing micronemes (in) and osmiophilic granular body embraced by a mitochondrion (bold arrow) (N - nucleus). Fig. 10. Microgametocyte (general view). (Bold arrow - electron-dense aggregate, $\mathrm{x}$ - vacuole). Scale bars $=0.5 \mu \mathrm{m}$. 
nucleolemma (Fig. 1). In the more differentiated uninucleate trophozoite a pellicle was formed by the addition of a thick inner bilaminate membrane which appeared as chain of fragments. The vague outline of a fine outer membrane could be traced between the outer thick wall and the erythrocyte body, with additional clefts and a mitochondrion within the cytoplasm of the red blood cell (Fig. 2). The parasite cytoplasm contained a large tubular mitochondrion and a few vacuoles with electron-dense debris (apparently pigment residue). An elongate cisterna seemed to extend from the nucleo-lemmal space (Fig. 2).

\section{Merogony}

Nuclear division coincided with the emergence of the merozoite primordia (Figs. 3, 4), leading to the gradual separation of the merozoites from the parent meront (Figs. 5-9). Beneath the wall-membrane of the parent meront, the merozoite primordium became invested by a pellicle which included an outer membrane and an inner double-layered membrane of fragmented appearance which emerged from an ER junction element (Figs. 3, 4). The nucleus of the parasite expanded into the base of the emerging merozoites and a presumed centriolar plaque occurred adjacent to one end of the nucleus (Fig. 4): microtubules occurred beneath the wall layer (Figs. 5, 8). The surfacing merozoites contained a large electron-dense body, which was filled with a dense granular matrix (Fig. 3): the contents of this body remained intact in the differentiating merozoites, or underwent further condensation (Figs. 4-6), or disin-tegration (Figs. 7, 8). In the latter event, vacuoles containing the granular matrix also occur in the parasitophorous vacuole (PV), alongside the merozoites (Fig. 7). In some of the forming merozoites the large electron-dense body (intact or disintegrating) was seen to be embraced by a tubular mitochondrion with a dense matrix (Figs. 6, 9). In the fully developed merozoite the inner layer of the pellicle retained, in part, its fragmented appearance (Figs. 7-9); beneath it occurred the regularly spaced microtubules. A distinct cytostome was seen in some merozoites (Fig. 7), and one was seen to possess an osmiophilic thickening of the inner pellicular membrane in the preapical zone (Fig. 6). The nucleus of the merozoite contains chromatin which was either homogenous (Fig. 5) or aggregated (Fig. 7). Micronemes began to form at the apical end, and became more conspicuous in fully differentiated merozoites (Fig. 9). The residuum of the meront formed a large food vacuole which contained pigment granules (Figs. 5, 6). Fully differentiated merozoites, bounded by a pellicle, and the meront's residuum remained enclosed inside a common membrane within the PV in the erythrocyte (Fig. 9). The enclosed space was filled with either a scattered flocculent substance or dense granular material (Figs. 6, 9).

\section{Microgametocyte}

Microgametocytes (Figs. 10, 11) measured approximately $5.2 \times 2.5 \mu \mathrm{m}$ ( 2 measured $)$ and were bounded by a double membrane which was closely applied to the PV wall which became detached during processing. The planes of the images studied failed to show a nucleus, although a small membrane-bounded inclusion was seen and possibly represented a segment of the nucleus. The large vacuole seen in Fig. 10 is either a food vacuole or the result of a processing fault: it was absent in other microgametocyte images. There were several osmiophilic bodies, some seen with their draining duct (Fig. 11); the cytoplasm was sparsely filled with ribosomes, but there were few organelles other than an electrondense, granular aggregate (Fig. 10) and a few ER elements (Fig. 11).

\section{Macrogametocytes}

Macrogametocytes (Figs. 12-14) were $4.5 \times 2.5 \mu \mathrm{m}$ in size ( 4 measured) and bounded by a wall composed of two membranes, and the closely applied, detached (due to processing) PV wall. The cytoplasm beneath was also bordered in part by ER elements and in part by a limiting membrane. More ER elements occured in the cytoplasm, where they also formed a large complex of tubuli and cisternae (Figs. 12, 14). The cytoplasm was densely packed with ribosomes, and also contained several electron-dense bodies, apparently osmiophilic bodies, one large tubular mitochondrion and one or two bound vesicles, including food vacuoles containing pigment granules (Figs. 12-14); the nucleus was not seen in any of the images obtained.

\section{DISCUSSION}

With the knowledge that mixed infections of Plasmodium kentropyxi and the $P$. tropiduri-like plasmodium commonly occurred in Kentropyx calcarata (Lainson et al. 2001), particular care was taken to select a lizard for our TEM study which showed only the latter parasite in Giemsa-stained blood films.

Although the general ultrastructure of the $P$. tropiduri-like parasite conforms with that of other reptilian and avian plasmodia (Aikawa and Jordan 1968, Aikawa 1971, Moore and Sinden 1974) the TEM images presented here do not closely resemble those described for P. tropiduri sensu stricto by Scorza (1971): possibly, therefore, we may be dealing with a different parasite. As previously discussed (Lainson et al. 2001), until more precise methods can be applied to separate those parasites showing such closely similar light microscopic morphology, we prefer to refer to the organisms of the present study as " $P$. tropiduri-like" (sensu Telford 1979). 
In all developmental stages of the Haemosporina studied by TEM, the outermost wall membrane is that

of the PV boundary, which becomes detached in
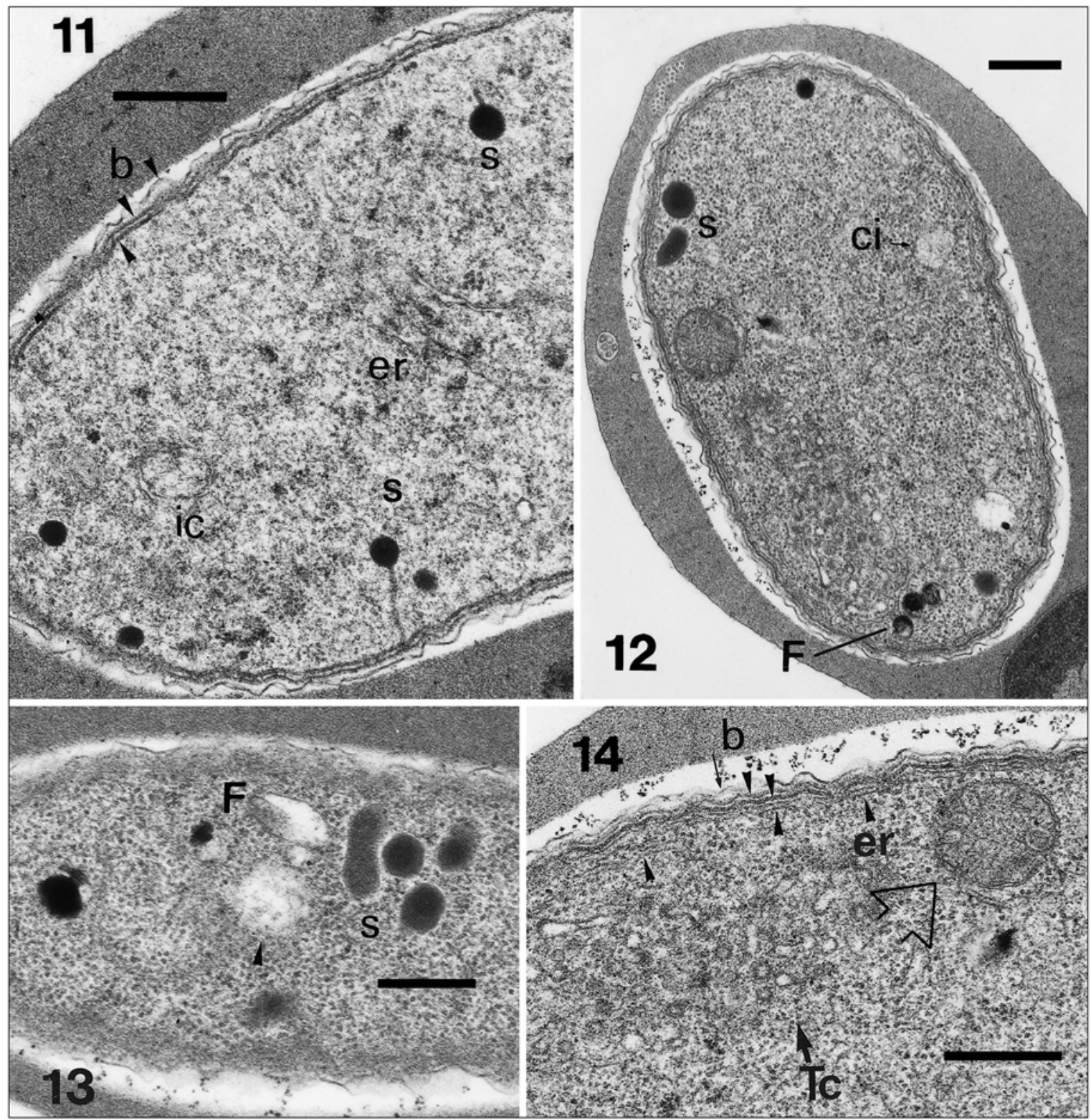

Figs. 11-14. Gametocytes of a Plasmodium tropiduri-like parasite of the lizard Kentropyx calcarata. Fig. 11. Enlarged view of Fig. 10: (b plus small arrowheads - the outer [host] and two inner [parasite] membranes, er - endoplasmic reticulum, ic bordered inclusions, s - osmiophilic bodies). Figs. 12, 13. Macrogametocytes. Fig. 14. Enlarged view of Fig. 12. Abbreviations for Figs. 12-14: b plus arrowheads - wall membranes, ci - cisterna, er plus arrowheads - endoplasmic reticulum complex beneath the cell wall, $\mathrm{F}$ - food vacuole with pigment, $\mathrm{s}$ - osmiophilic bodies connected to ductules, Tc - a complex of tubuli and cisternae, $\mathrm{v}$ - vesicle, open arrow - mitochondrion. Scale bars $=0.5 \mu \mathrm{m}$.

processing (Aikawa 1971). Our image of the cytoplasm of the trophozoite-infected erythrocyte revealed a mitochondrion as well as cytoplasmic clefts. The latter, reported in infections by Plasmodium falciparum and $P$. malariae, have been suggested to be associated with the transport of specific malarial antigens from the parasite to the erythrocyte membrane skeleton (Atkinson and Aikawa 1990).

Both merozoites and gametocytes of reptilian and avian species of Plasmodium have been shown to be bound by three membranes, of which the outer (PV) and the innermost are the thickest: the middle membrane 
can be very fine (Aikawa and Jordan 1968, Aikawa et al. 1969). The number and configuration of the wall membranes of Haemoproteus gametocytes have been shown to be similar, although varying in their relative

thickness, (Paperna and Boulard 2000). The two inner membranes of the gametocytes of the $P$. tropiduri-like parasite of the present study were of equal thickness.

In emerging merozoites the innermost, thick membrane was interrupted in a manner similar to that seen in other reptilian and avian Plasmodium species (Aikawa 1967, Aikawa and Jordan 1968, Scorza 1971). It has been shown, by negative staining without sectioning, that this wall is a continuous labyrinthine structure which, together with the microtubules, may serve as a cytoskeleton which provides rigidity for the merozoite (Aikawa 1967). Breaking down of the labyrinthine wall occurs, as in other plasmodian merozoites, on entry into a new host cell (Aikawa and Jordan 1968), and the observed trophozoites remain bounded only by a single thin membrane, applied to the membrane of host origin; groups of microtubules, however, are retained. The inner labyrinthine complex appears to be already reassembled at the trophozoite stage, prior to subsequent merogony.

In the process of meront division, the merozoite rudiment begins differentiating from an extension of the expanding parent nucleus. The wall extends from a juncture formed at the eventual separation point of the merozoite nucleus. A suggestion of this process is seen in TEM images of Plasmodium elongatum (Aikawa 1971).

We remain uncertain of the identity, and hence the function, of the large, electron-dense bodies with the unique consistency of osmiophilic grains, seen in emerging merozoites. It is not certain if they are anlagen of paired organelles; they were seen at some stage to be disintegrating, or evicted into the PV lumen, a phenomenon that has not previously been reported in descriptions of Plasmodium species. In some merozoites these electron-dense bodies become apposed by the large mitochondrion with a dense matrix, in a similar manner as the spherical bodies (see Aikawa 1971) in erythrocytic merozoites of avian (Aikawa 1966) and reptilian (Aikawa and Jordan 1968, Scorza 1971) Plasmodium spp.

Following merozoite formation, the meront residuum remains the site of a large food vacuole, as has previously been seen in images of $P$. elongatum (Aikawa et al. 1969) and P. mexicanum (Moore and Sinden 1974). As is the case of $P$. floridense (Aikawa and Jordan 1968) and P. tropiduri (Scorza 1971), emerging merozoites of the P. tropiduri-like parasite are equipped with a large, conspicuous cytostome. Cytostomes, usually demonstrated in gametocyte images (Aikawa et al. 1969), were not seen in the presently described gametocytes.

The large mitochondria seen in macrogametocytes in the present study are closely reminiscent of those having an "electron-opaque" matrix, seen in $P$. gallinaceum and P. elongatum (Aikawa et al. 1969) and P. tropiduri (Scorza 1971). In asexual stages studied here (trophozoites and meronts), the matrix becomes heavily granular or even electron-dense in consistency.

The number and size of the osmiophilic bodies has been shown to vary considerably among images of gametocytes of Plasmodium spp. (Aikawa et al. 1969, Scorza 1971) and of Haemoproteus (Paperna and Boulard 2000). In the latter they are more numerous in macrogametocytes than in microgametocytes of the same species.

In our light microscope study (Lainson et al. 2001) pigment granules were detectable in most parasites in the Giemsa-stained blood films viewed by polarised light, particularly in the more advanced asexual stages and mature gametocytes. Few pigment aggregates were detected in the TEM images; they were conspicuous only in the meront residuum and in its food vacuole. Scarcity of pigment aggregations could be due either to processing or to the early nature of the infection used for this ultrastructural study.

\section{REFERENCES}

AIKAWA M. 1966: The fine structure of the erythrocytic stages of three avian malarial parasites, Plasmodium fallax, P. lophurae and P. cathemerium. Am. J. Trop. Med. Hyg. 15: 449-471.

AIKAWA M. 1967: Ultrastructure of the pellicular complex of Plasmodium fallax. J. Cell Biol. 35: 103-113.

AIKAWA M. 1971: Plasmodium: the fine structure of malaria parasites. Exp. Parasitol. 30: 284-320.

AIKAWA M., HUFF C.G., SPRINZ H. 1969: Comparative fine structure of the gametocytes of avian, reptilian and mammalian malarial parasites. J. Ultrastruct. Res. 26: 316331.
AIKAWA M., JORDAN H.B. 1968: Fine structure of a reptilian malarial parasite. J. Parasitol. 54: 1023-1033.

ARAGÃO H. de B., NEIVA A. 1909: A contribution to the study of the intraglobular parasites of the lizards. Two new species of Plasmodium. Pl. diploglossi n. sp. and Pl. tropiduri, n. sp. Mem. Inst. Oswaldo Cruz 1: 44-50.

ATKINSON C.T., AIKAWA M. 1990: Ultrastructure of malaria-infected erythrocytes. Blood Cells 16: 351-368.

LAINSON R., LANDAU I., PAPERNA I. 2001: Plasmodium kentropyxi n. sp. (Apicomplexa: Haemosporina: Plasmodiidae) and a Plasmodium tropiduri-like parasite in the lizard Kentropyx calcarata (Lacertilia: Teiidae) in north Brazil. Parasite 8: 107-113. 
LAINSON R., SHAW J.J. 1969: New host records for Plasmodium diploglossi, P. tropiduri Argão \& Neiva, 1909, and P. cnemidophori Carini, 1941. Parasitology 59: 163-170.

MOORE J., SINDEN R.E. 1974: Fine structure of Plasmodium mexicanum. J. Parasitol. 60: 825-833.

PAPERNA I., BOULARD Y. 2000: Fine structure of gametocytes in five species of Haemoproteus (Haemosporidia) from geckoes and agamid lizards. Zoosystema 22: 443457.

Received 16 March 2001
SCORZA J.V. 1971: Electron microscope study of the blood stages of Plasmodium tropiduri, a lizard malaria parasite. Parasitology 63: 1-20.

TELFORD S.R. 1979: A taxonomic consideration of some Plasmodium species from iguanid lizards. Ann. Parasitol. Hum. Comp. 54: 129-144.

WALLIKER D. 1966: Malaria parasites of some Brazilian lizards. Parasitology 56: 39-44.

Accepted 13 June 2001 